\title{
Immuno-oncology safety education experience: Key lessons from ipilimumab (IPI)
}

\author{
Heddy Bartell ${ }^{1 *}$, Jedd Wolchok ${ }^{2}$, F Stephen Hodi ${ }^{3}$, Helen Liư ${ }^{4}$, Cynthia Wojtaszek ${ }^{5}$, Jeffrey Weber ${ }^{6}$ \\ From 30th Annual Meeting and Associated Programs of the Society for Immunotherapy of Cancer (SITC 2015) \\ National Harbor, MD, USA. 4-8 November 2015
}

\begin{abstract}
Background
IPI (Yervoy ${ }^{\circledR}$, Bristol-Myers Squibb) blocks cytotoxic Tlymphocyte antigen-4, a regulatory molecule on activated T cells. In March 2011, IPI, indicated for unresectable or metastatic melanoma (MEL), became the first immune checkpoint inhibitor approved by the US Food and Drug Administration (FDA). Early in development, severe and fatal immune-related adverse events (irAEs) emerged and were attributed to IPI's mechanism of action, occurring mostly during treatment, although a minority occurred weeks to months after drug discontinuation. irAE management guidelines created in collaboration with external experts and study investigators were progressively incorporated into the IPI Investigator's Brochure.
\end{abstract}

\section{Methods}

At approval, the FDA stipulated that a Risk Evaluation and Mitigation Strategy (REMS) communication plan directed at healthcare providers (HCPs) be developed to ensure that benefits with IPI outweighed irAE risks. The REMS goal was to inform HCPs about serious risks with IPI and irAE management. Multichannel educational outreach to HCPs was implemented at launch and every 6 months for 3 years, in addition to a dedicated webpage and triggered outreach to new prescribers. REMS materials included a Dear HCP Letter, management guide, nursing checklist, and patient (pt) wallet card. Audience was HCPs who manage pts with MEL (eg, oncologists) and HCPs who may be consulted about irAEs (eg, gastroenterologists). Professional medical societies were also contacted to inform their memberships.

\section{Results}

During the 3-year outreach effort, REMS materials were distributed via $>160,000-250,000$ emails per outreach

${ }^{1}$ Bristol-Myers Squibb, Plainsboro, NJ, USA

Full list of author information is available at the end of the article and $>700,000$ total mailings. Prescriber understanding of irAE management was assessed twice, via oncologist knowledge surveys, with median correct scores of $76 \%$ in 2012 and $85 \%$ in 2014. Academic and community HCPs tested equally well. Pharmacovigilance review of fatal irAEs showed no delay in recognizing and treating irAEs, and management was consistent with US prescribing information. Periodic, aggregate safety data did not identify any new safety concerns. Because the REMS met its goal and key activities were successfully completed with no required modifications at the 18-month and the 3-year assessments, the FDA released the REMS in March 2015. Voluntary IPI safety education efforts continue worldwide.

\section{Conclusions}

Management algorithms and safety education materials were created through early collaboration with investigators and then broadly disseminated through the IPI REMS, increasing HCP awareness of irAE management. Socialization of irAE identification and management through the sentinel IPI safety education experience has benefited newer immuno-oncology agents, and IPI safety materials remain highly regarded and utilized by HCPs.

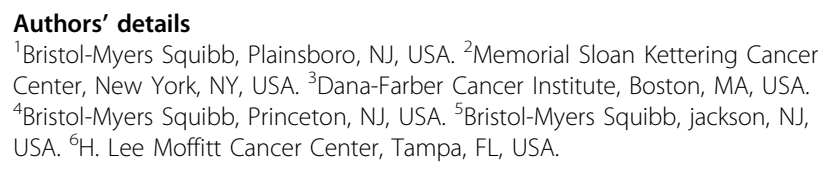
Center, New York, NY, USA. ${ }^{3}$ Dana-Farber Cancer Institute, Boston, MA, USA. ${ }^{4}$ Bristol-Myers Squibb, Princeton, NJ, USA. ${ }^{5}$ Bristol-Myers Squibb, jackson, NJ, USA. ${ }^{6} \mathrm{H}$. Lee Moffitt Cancer Center, Tampa, FL, USA.

Published: 4 November 2015 